\title{
Bolide meteor streams
}

\section{Štefan Gajdoš and Vladimir Porubčan}

\author{
Dept. of Astronomy, Physics of the Earth, and Meteorology \\ Faculty of Mathematics, Physics and Informatics \\ Comenius University Bratislava, Slovakia \\ email: gajdos@fmph.uniba.sk
}

\begin{abstract}
The current version of the IAU Meteor Data Centre catalogue of photographic orbits comprising the orbital and geophysical data of 4581 meteors is applied for a more representative search of the mean orbits of bolide meteor streams among photographic meteors. We have made the search based on a computerized stream search procedure utilizing the Southworth-Hawkins D-criterion. The results are compared with the previous similar analysis made by Porubčan \& Gavajdová (1994).
\end{abstract}

Keywords. Meteors, meteoroids, bolide meteor streams.

\section{Introduction}

Porubčan \& Gavajdová (1994) published a list of fireball meteoroid streams compiled from the IAU Meteor Data Centre catalogue of photographic orbits (Lindblad 1991) based on a set of 1028 fireballs $\left(M_{p h} \leqslant-3^{m}\right)$. They found 30 fireball streams among known meteoroid streams of which 23 streams belong to regular streams listed in Cook (1973) or Kronk (1988) including their northern and southern branches and further they found additional 19 new fireball streams. As an subsidiary result of their analysis was that less than a half (46\%) of the fireball population belongs to meteoroid streams population.

In our present analysis we have searched for potential fireball streams utilizing an updated version of the IAU MDC catalogue (Lindblad, Neslušan, Porubčan, et al. (2004)) of precise photographic meteor orbits summarizing a total of 4581 meteors. The catalogue contains a total of 1541 bolides having the $\left(M_{p h} \leqslant-3^{m}\right)$, i.e. additional 513 bolides with respect to the previous search made by Porubčan \& Gavajdová (1994). This means a substantial increase of data which can enable to define more precise mean orbits of the streams and detect additional members of some minor streams or associations.

Statistics of data available for analysis is summarized in Table 1, which lists the numbers of bolides observed in individual months of the year. As evident, the most numerous data are from August (Perseids) followed by data from autumn months, October, November and December. Table 1 shows an inhomogeneous distribution of observations throughout the year resulting from obervational campaigns carried out predominantly during the activity of major meteor showers. The last column of Table 1 lists the number of meteors without an estimate of brightness being available. The largest number of such observations is from January (Quadrantids), August (Perseids) and December (Geminids).

Of the 4581 photographic meteors listed in the current version of the IAU MDC catalogue, 1563 meteors are without an estimation of the brightness $\left(M_{p h}\right)$. Therefore, only 1541 bolides with $M_{p h} \leqslant-3^{m}$ could be selected from the catalogue. Table 2 shows the statistics of various brightness groups of fireballs listed in the catalogue. 
Table 1. The numbers of bolides recorded in individual months. The first column gives number of meteors brighter (or equal) $-3^{m}$, while the second one gives number of bolides, according commonly used brightness limit $\left(-4^{m}\right)$. The third column contains meteors fainter than $-3^{m}$. The last column lists the number of meteors having no magnitude estimation - there are "no data" on their brightness available.

\begin{tabular}{ccccc}
\hline Months & $M_{p h} \leqslant-3^{m}$ & $M_{p h} \leqslant-4^{m}$ & $M_{p h}>-3^{m}$ & no data \\
\hline Jan & 82 & 80 & 63 & 79 \\
Feb & 63 & 61 & 37 & 54 \\
Mar & 65 & 60 & 73 & 26 \\
Apr & 69 & 62 & 91 & 27 \\
May & 62 & 56 & 64 & 60 \\
Jun & 47 & 40 & 81 & 51 \\
Jul & 106 & 76 & 102 & 73 \\
Aug & 524 & 354 & 408 & 460 \\
Sep & 93 & 73 & 99 & 140 \\
Oct & 171 & 147 & 143 & 147 \\
Nov & 130 & 115 & 76 & 110 \\
Dec & 129 & 107 & 240 & 336 \\
\hline SUM & 1541 & 1231 & 1477 & 1563 \\
\hline
\end{tabular}

Table 2. The numbers of bolides recorded up to other limits of the absolute photographic magnitude $M_{p h}$.

\begin{tabular}{cc}
\hline$M_{p h}$ limit & Number \\
\hline$M_{p h} \leqslant-5^{m}$ & 997 \\
$M_{p h} \leqslant-7^{m}$ & 693 \\
$M_{p h} \leqslant-9^{m}$ & 369 \\
$M_{p h} \leqslant-11^{m}$ & 128 \\
\hline
\end{tabular}

\section{Analysis}

We have made a search for new members of the streams from the updated list of bolides in order to derive more precise mean orbits of the streams.

The analysis was based on a computerized stream search procedure utilizing the Southworth-Hawkins D-criterion (Southworth \& Hawkins 1963) and an iteration procedure (Porubčan \& Gavajdová 1994). For the analysis a limiting value of $D \leqslant 0.20$ was applied. This value is stricter than the value applied by Porubčan \& Gavajdová (1994) in their search for fireball streams, where they have set $D \leqslant 0.25$ for the whole set of data.

The results obtained in our search are listed in Table 3 and 4, where the arithmetic mean values of the orbital elements together with the geocentric radiants and geocentric velocities are presented. The last two columns list the number of individual members of the streams obtained by Porubčan \& Gavajdová (1994) and in this analysis, respectively. The largest increase of stream members is for the Perseids and Geminids as the best monitored meteor streams by almost all photographic observational campaigns.

The investigation has shown that some ecliptic low-inclination and dispersed streams (as Taurids) cannot be separated distinctly from the background and that some of their members appear also in smaller streams active either parallel or close to these streams. To separate them unequivocally the limiting $D$ value has to be lower and carefully selected for each stream individually. 
Table 3. Mean orbital parameters, geocentric velocity (in $\mathrm{km} / \mathrm{s}$ ) and radiants of the known meteoroid streams derived from bolides of $M_{p h} \leqslant-3^{m}$. In some cases, * denotes the showers, where a lower limit $D \leqslant 0.15$ had to be applied for the separation of the stream.

\begin{tabular}{|c|c|c|c|c|c|c|c|c|c|c|c|c|}
\hline Shower & $q$ & $a$ & $e$ & $i$ & $\omega$ & $\Omega$ & $\pi$ & $V_{g}$ & R.A. & Dec & $N_{1}$ & $N_{2}$ \\
\hline Que & 977 & 3.122 & 686 & 72.3 & 170.7 & 284.8 & 95.5 & 41.5 & 231 & 48.6 & 6 & \\
\hline Leor & & & 634 & 0 & 32.0 & 45.7 & 217.8 & 14.4 & 150 & 22.7 & & \\
\hline Virgi & 387 & 46 & 914 & 3.8 & 86.5 & 348.5 & 275.0 & 31.0 & 178.1 & & & \\
\hline$\pi$ Virginids & 618 & 2.257 & 0.728 & 4.8 & 64.6 & 16.9 & 281.5 & 21.5 & 195.8 & 0.8 & & 7 \\
\hline$\sigma$ Leonids (S) & 754 & 1.968 & 0.620 & 4.5 & 68.8 & 197.1 & 266.0 & 16.5 & 181.5 & -10.7 & & 8 \\
\hline Lyrids & 0.925 & 66.509 & 0.986 & 79.8 & 212.8 & 32.7 & 245.5 & 47.0 & 272.8 & 33.7 & & 9 \\
\hline$\alpha \mathrm{Scc}$ & & & 0.87 & & 117.3 & 237.5 & 354.8 & 31.0 & 247.1 & -28.7 & 3 & 3 \\
\hline$\theta \mathrm{Op}$ & 566 & 2.367 & 0.761 & 4.5 & 271.4 & 92.2 & 3.6 & 22.9 & 272.4 & -17.3 & 6 & \\
\hline$\theta \mathrm{Oph}$ & 657 & 1.949 & 0.660 & 2.7 & 83.0 & 285.4 & 8.4 & 18.9 & 282.4 & -27.3 & & \\
\hline$\iota$ Aque & 238 & 3.5 & 0.929 & 5.8 & 306.6 & 130.0 & 76.6 & 34.4 & 327.5 & -9.3 & 3 & 3 \\
\hline$\alpha$ Cap & 589 & 2.42 & 0.7 & 8.2 & 268.3 & 128.1 & & 22.5 & 307.3 & -8.1 & 15 & 27 \\
\hline$\alpha$ Capı & 0.519 & 2.149 & 0.762 & 4.0 & 97.2 & 327.0 & & 23.2 & 334.0 & -15.6 & 6 & 5 \\
\hline$\delta$ Aquarids $(\mathrm{N})$ & 0.085 & & 0.971 & 20.0 & 329.3 & 136.9 & & 40.7 & 342.7 & -1 & 5 & 6 \\
\hline$\delta \mathrm{A}$ & & 3. & 0.96 & 26.5 & 147.3 & 313.1 & 10 & 40.6 & 344.4 & -15.2 & 10 & 11 \\
\hline Pers & 951 & 27.396 & 0.965 & 112.9 & 151.1 & 138.7 & 289. & 59.2 & 46.0 & 57.9 & 193 & *230 \\
\hline Pers & & & 0.542 & 8.3 & 6 & 137.2 & 27 & 53.1 & 45.0 & 57 & 6 & 4 \\
\hline$\kappa$ Cygnids & 0.983 & 3.535 & 0.721 & 32.7 & 200.0 & 141.6 & 341.7 & 21.6 & 279.5 & 50.8 & 13 & 21 \\
\hline Sept. Perseids & 0.734 & -44.510 & 1.016 & 140.6 & 242.4 & 166.9 & 49.4 & 65.6 & 47.4 & 39.0 & 3 & 3 \\
\hline Piscids (S) & 0.544 & & 0.844 & 2.2 & 90.0 & 12.2 & 102.3 & 24.4 & 12.0 & 2.4 & 7 & 6 \\
\hline Andr & & & 0.760 & 5 & 264.2 & 189.9 & & 21.3 & 4.0 & 10.2 & 5 & 6 \\
\hline & & & 0.679 & & 245 & 07.9 & & 18.1 & 3.9 & 31 & 3 & 3 \\
\hline & 0 & 338 & 0.575 & 22.9 & 180.6 & 203.0 & 23.6 & 15.4 & 278.5 & 48. & 7 & 12 \\
\hline Taur & 33 & .071 & 0.839 & 2.8 & 297.3 & 222.8 & 160 & 28.8 & 53.1 & 21.4 & 12 & $* 11$ \\
\hline Taurids (S) & 0.364 & 2.232 & 0.836 & 5.0 & 112.8 & 43.6 & 156.4 & 28.2 & 53.7 & 14.7 & 19 & $* 27$ \\
\hline Orionids & 0.578 & 27.478 & 0.978 & 163.7 & 81.2 & 27.8 & 109.0 & 66.7 & 94.5 & 15.6 & 12 & 17 \\
\hline & 0.985 & & 0.894 & 162.6 & 174.5 & 236.0 & & 70.6 & 153.8 & 21.7 & 5 & 9 \\
\hline & & & 0.994 & 128.1 & 118.3 & 77.4 & & 59.2 & 126.2 & & 4 & 2 \\
\hline & & & & 2.5 & 285 & 255.8 & & 26.1 & 83.1 & 25. & 7 & \\
\hline & & & & & & 75.3 & & 23.4 & 77.6 & 16. & 6 & $*_{5}$ \\
\hline Geminids & 0.138 & 1.375 & 0.899 & 24.1 & 324.6 & 261.7 & 226.3 & 34.7 & 113.2 & 32.3 & 19 & 38 \\
\hline
\end{tabular}

Fig. 1 shows the distribution of the radiants (right ascension and declination) of all bolides and the mean stream radiants listed in Tables 3 and 4, respectively.

\section{Discussion}

Based on the updated version of the IAU MDC catalogue of photographic orbit, we have derived more precise orbits of fireball meteor streams (meteors with the absolute photographic magnitude $M_{p h} \leqslant-3^{m}$ ) by applying a limiting value of $D \leqslant 0.20$ to all the streams. The search has shown that each stream has to be analyzed individually and equal limiting value of $D$ can be used only as a first approximation.

The statistics of meteors in the catalogue shows that the distribution of meteors all over the year is not homogeneous, while the Perseids are by far the richest stream in the majority of the photographic catalogues. This fact is due to a selection effect caused by observations carried out predominantly in the summer months. Eliminating the Perseids, the distribution of fireballs in the IAU MDC catalogue is more homogeneous. For the majority of the known meteor streams an increase in the shower numbers is recorded (see Table 3). On the contrary, in some cases a decrease in the number of members is 
Table 4. Mean orbital parameters, geocentric velocity and radiants of minor bolide streams and their number $N_{1}$, as stated by Porubčan \& Gavajdová (1994), and number $N_{2}$ resulting from our analysis.

\begin{tabular}{lcccrrrrrrrrr}
\hline \multicolumn{1}{c}{ Shower } & $q$ & $a$ & $e$ & \multicolumn{1}{c}{$i$} & \multicolumn{1}{c}{$\omega$} & \multicolumn{1}{c}{$\Omega$} & \multicolumn{1}{c}{$\pi$} & \multicolumn{1}{c}{$V_{g}$} & R.A. & Dec & $N_{1}$ & $N_{2}$ \\
\hline$\alpha$ Cancrids & 0.482 & 1.160 & 0.582 & 7.2 & 113.0 & 124.1 & 237.1 & 19.3 & 135.7 & 6.2 & 3 & 3 \\
$\beta$ Cancrids & 0.800 & 2.115 & 0.614 & 4.1 & 59.9 & 136.1 & 196.0 & 14.8 & 120.0 & 10.9 & 3 & 5 \\
Lynxids & 0.966 & 1.925 & 0.493 & 6.9 & 202.4 & 343.4 & 185.9 & 8.9 & 124.7 & 49.3 & 3 & 4 \\
March Cassiopeids & 0.939 & 2.406 & 0.610 & 15.8 & 149.9 & 349.4 & 139.3 & 13.6 & 358.7 & 54.0 & 4 & 5 \\
$\alpha$ Coma Berenicids & 0.858 & 2.532 & 0.660 & 9.9 & 229.5 & 23.2 & 252.7 & 15.4 & 189.9 & 20.8 & 5 & 7 \\
$\gamma$ Corvids & 0.832 & 2.116 & 0.610 & 4.5 & 55.3 & 208.3 & 263.7 & 14.4 & 182.2 & -13.2 & 5 & 9 \\
$\beta$ Librids & 0.508 & 2.623 & 0.808 & 10.1 & 276.4 & 49.8 & 326.2 & 25.9 & 233.8 & -7.3 & 3 & 7 \\
$\eta$ Ursa Maiorids & 0.939 & 2.308 & 0.592 & 12.0 & 212.5 & 32.2 & 244.7 & 13.1 & 192.7 & 33.7 & 3 & 9 \\
$\mu$ Ursa Maiorids & 0.977 & 2.154 & 0.546 & 8.8 & 198.7 & 48.2 & 247.0 & 10.5 & 186.5 & 32.0 & 3 & 8 \\
$\lambda$ Aquilids & 0.943 & 1.840 & 0.485 & 4.1 & 215.8 & 150.7 & 6.5 & 9.2 & 290.4 & -5.7 & 4 & 6 \\
$\delta$ Piscids & 0.257 & 1.881 & 0.864 & 4.3 & 307.3 & 173.6 & 121.0 & 30.6 & 9.1 & 7.1 & 3 & 5 \\
Sept. $\iota$ Aquarids & 0.908 & 2.361 & 0.613 & 1.6 & 39.4 & 1.3 & 40.8 & 11.2 & 328.4 & -19.0 & 3 & 5 \\
$\lambda$ Cygnids & 0.947 & 2.532 & 0.626 & 11.2 & 206.7 & 202.7 & 49.5 & 12.1 & 321.7 & 23.8 & 3 & 7 \\
$\alpha$ Taurids & 0.792 & 2.205 & 0.641 & 3.0 & 60.0 & 85.3 & 145.3 & 14.9 & 67.9 & 14.8 & 4 & 12 \\
Dec. Aurigids & 0.665 & 2.153 & 0.692 & 7.2 & 257.7 & 270.7 & 168.5 & 19.5 & 85.5 & 35.5 & 5 & 5 \\
$\tau$ Geminids (N) & 0.385 & 1.446 & 0.734 & 4.0 & 296.4 & 272.8 & 209.2 & 24.5 & 108.3 & 26.5 & 4 & 4 \\
$\tau$ Geminids (S) & 0.536 & 2.036 & 0.733 & 4.6 & 94.2 & 100.8 & 195.0 & 22.8 & 104.1 & 16.7 & 6 & 6 \\
Dec. $\beta$ Perseids & 0.929 & 2.238 & 0.584 & 4.9 & 208.5 & 275.5 & 124.1 & 10.3 & 39.9 & 35.2 & 3 & 8 \\
\hline
\end{tabular}

revealed, mainly due to more rigorous limit for $D$ (e.g. narrow Perseids-2, $\sigma$ Hydrids, both the $\chi$ Orionids).

A representative stream search desires to take into account all the factors influencing geometry of encounter of the potential meteoroid streams with the Earth, allowing for the size and form of the radiant, its daily motion and dispersion of geocentric velocities of the stream members.

It is apparent that these factors strongly influence the possibility of detection, especially for meteoroid streams moving in low-inclination, typical asteroidal orbits and this affects the posibility of detection of meteoroid streams of asteroidal origin.

The Earth approaching asteroids are moving in prograde orbits with low geocentric velocities, then due to geometry of the encounter with the Earth their potential streams will be dispersed and large radiant areas; they frequently split into several "sub-streams" and this greatly hampers their detection and recognition as single meteoroid streams (Kresák 1968). Therefore the above factors have to be taken into account, at least in a study or in the selection of meteoroid streams moving in low inclination short-period orbits.

In a search for meteoroid stream and their parent bodies besides the orbital similarity also the orbital evolution of both the parent body and of the members of the stream has to be taken into account.

According to the principles of meteoroid stream formation (Babadzhanov 1996), meteor showers can be produced also by parents which are, at present, on orbits at a distance from the Earth's orbit $>0.3 \mathrm{AU}$, but which crossed it in the past. Therefore a study also of the orbital evolution of the stream members is desirable.

For comets as parent bodies of meteoroid streams, resonant binding of cometary dust particles provides a new possibility in the field of meteoroid stream research. As recently revealed, mean-motion resonance with Jupiter is responsible, at least in the Leonids and the June Bootids, for dust trails evolution (e.g. Asher, Bailey \& Emel'yanenko (1999), Asher \& Emel'yanenko (2002)). Also, a detailed study of dust filaments formation resulted 

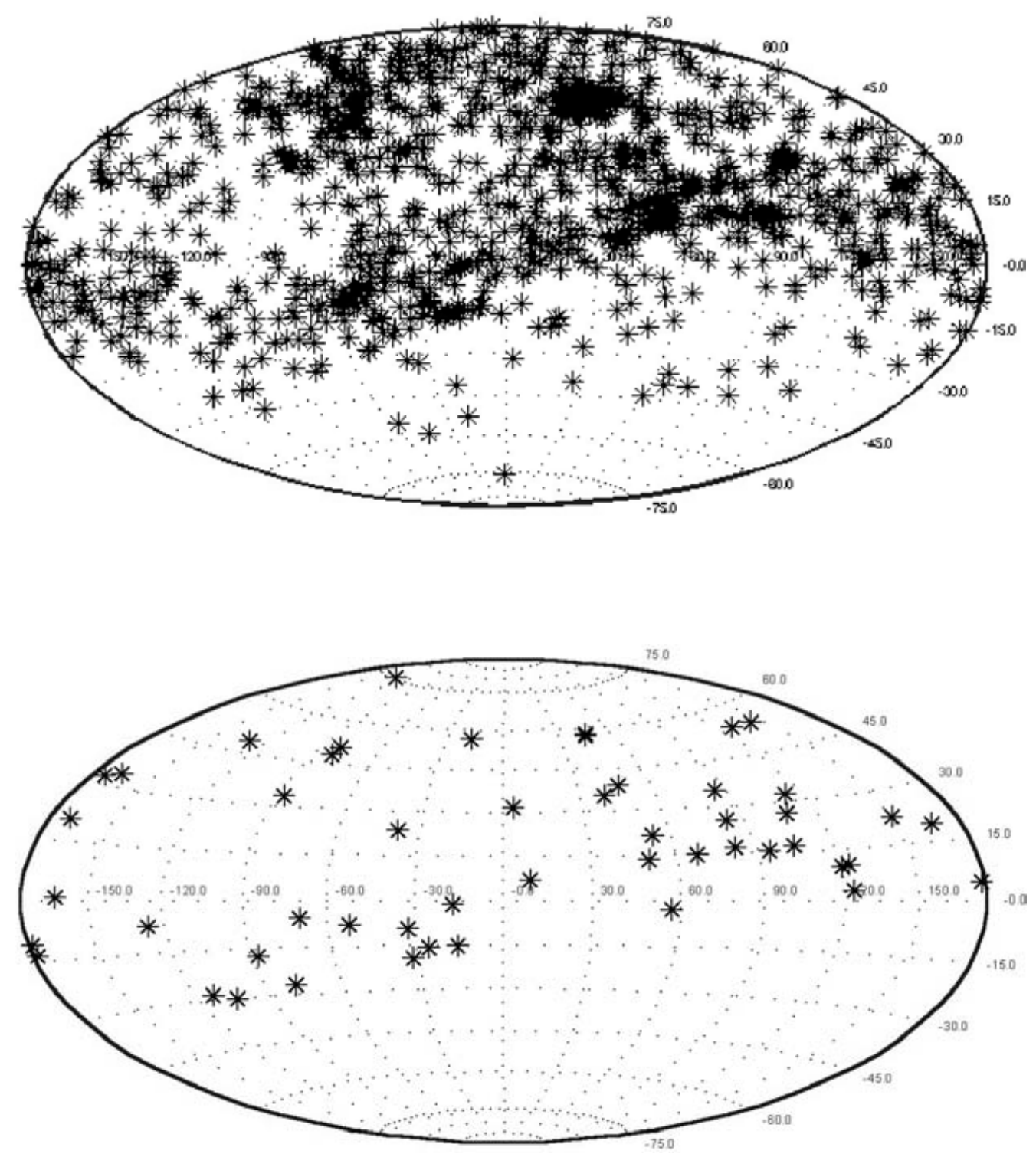

Figure 1.

Up - Distribution of radiants $(\alpha, \delta)$ of all 1541 bolides separated from the IAU MDC catalogue. Down - Distribution of the mean radiants $(\alpha, \delta)$ of the streams listed in Table 3 and 4.

in successful predictions of enhanced activity of the Leonids starting from the last years of the previous millennium (Asher 1999; Lyytinen 1999). At the same time, as during recent returns of Leonids rich in bolides, especially in 1998, many new orbits were obtained, a great increase in the number of Leonid orbits also in photographic catalogues is to be expected rather soon.

\section{Acknowledgements}

The authors acknowledge support of the research by the VEGA grants Nos. 01/0204/03 and $01 / 0206 / 03$. 


\section{References}

Asher, D.J. 1999, Mon. Not. R. Astron. Soc. 307, 919

Asher, D.J., Bailey, M.E. \& Emel'yanenko, V.V. 1999, Mon. Not. R. Astron. Soc. 304, L53

Asher, D.J. \& Emel'yanenko, V.V. 2002, Mon. Not. R. Astron. Soc. 331, 126

Babadzhanov, P.B. 1996, Sol. Sys. Res. 30, 499

Cook, A.F. 1973, Working List of Meteor Streams, NASA SP 319, 183

Kresák, L. 1968, in: P.M. Millman and L. Kresák (eds.), Physics and Dynamics of Meteors, (Dordrecht: D. Reidel Publ. Co.), p. 391

Kronk, G.W. 1988, Meteor Showers: A Descriptive Catalogue, (Enslow Publishers, Hillside, USA), p. 291

Lindblad, B.A. 1991, in: A.C. Levasseur-Regourd and H.Hasegawa (eds.), Origin and Evolution of Interplanetary Dust, (Dordrecht: Kluwer Academic Publishers), p. 311

Lindblad, B.A., Neslušan, L., Porubčan, V., Svoreň, J. 2004, Earth, Moon, Planets, in press Lyytinen, E. 1999, Meta Res. Bull. 8, 33

Porubčan, V. \& Gavajdová, M. 1994, Planet. Space Sci. 42, 151

Southworth, R.B. \& Hawkins, G.S. 1963, Smithson. Contrib. Astrophys. 7, 261 